\section{Urgency-Adjusted Utilization of EMS by Emergency Department Patients}

\section{Suter RE, Suter BS \\ Department of Emergency Medicine, Brooke Army Medical Center San Antonio, Texas, USA}

Purpose: Patients presenting to Emergency Departments (ED) may or may not access the EMS system as a means of transportation. This study sought to identify patterns of EMS utilization so that the design of interventions to encourage appropriate utilization of EMS by ED patients can be facilitated.

Design and Methods: Descriptive, consecutive sample derived from all 10,460 patients entering an academic ED over a threemonth period. Inclusion criteria were arrival by EMS or triage as urgent or emergent. Patients were excluded if EMS transport primarily was for non-ambulatory/institutionalized status. All eligible subjects were enrolled $(n=1485)$.

Main Results: EMS transportation was utilized by $37 \%$ of the subjects. Using triage criteria, $16 \%$ of EMS transports were deemed inappropriate. Converse!y, 67\% of urgent and emergent subjects did not use EMS transportation. Subjects with significantly higher rates of EMS use were those age $40-59$ years and those with neurologic or traumatic complaints $(p<.05)$. Significantly lower rates of EMS utilization were found in subjects age 10-19 years and those with cardiac complaints $(p<.05)$. Inappropriate EMS use was highest in subjects age $40-59$ years $(p<.05)$.

Conclusion: EMS transportation probably is underutilized on an overall basis. There is wide variability of use which cannot be explained using simple appropriateness criteria alone. The demand on EMS resources changes with population demographics. Efforts should be made to improve EMS utilization, especially for patients with cardiac complaints.

\section{On-Line Medical Command and Patient- Initiated Refusals of Prehospital Care: Patient Outcome and Ambulance Call Report Documentation}

\author{
Cone DC, Kim DT, Davidson SJ \\ Department of Emergency Medicine, \\ The Medical College of Pennsylvania, \\ Philadelphia, Pennsylvania, USA
}

Hypothesis: On-line medical command (OLMC) for patientinitiated refusal of care (PIR) is associated with improved ambulance call report (ACR) documentation. Patients who refuse care may be ill and may require hospitalization.

Methods: All PIR from May 1992 to October 1992 was identified retrospectively for a suburban, hospital-based advanced life support service with 4,200 annual responses. Policy requires OLMC prior to release of a patient refusing care. Each ACR was examined by two authors to determine adequacy of documentation (presence of vital signs, physical exam, mental status/orientation, patient not a minor or suicidal). Patients were contacted by telephone to determine outcome. Admission diagnosis was obtained for admitted patients.

Results: Eighty-one PIR were identified during the study period. In $58(72 \%)$ PIR, OLMC was not established. In 25 (43\%) of these, reviewers found insufficient ACR documentation. Of the 23 (28\%) PIR with OLMC, only two (9\%) had insufficient documentation ( $p<.001$, Fisher's exact test). Of the $54(67 \%)$ PIR with follow-up, $46(85 \%)$ sought no further care and did well. One was later seen in an ED and discharged, four $(7 \%)$ were admitted to non-critical settings, and three $(5 \%)$ were admitted to monitored units, for an overall admission rate of $13 \%$. There were no known deaths.

Conclusions: The ACR documentation of PIR is better with OLMC than without. Further study may elucidate a role for OLMC in preventing PIR by incompetent patients, convincing competent but apparently ill patients who intentionally refused care to accept transport, or assisting paramedics with difficult circumstances of PIR. 